\title{
Evaluation of high-resolution microarray platforms for genomic profiling of bone tumours
}

\author{
Stine H Kresse ${ }^{1}$, Karoly Szuhai ${ }^{2}$, Ana H Barragan-Polania ${ }^{1,3}$, Halfdan Rydbeck ${ }^{1,4}$, Anne-Marie Cleton-Jansen ${ }^{5}$, \\ Ola Myklebost ${ }^{1,3}$, Leonardo A Meza-Zepeda ${ }^{1,3^{*}}$
}

\begin{abstract}
Background: Several high-density oligonucleotide microarray platforms are available for genome-wide single nucleotide polymorphism (SNP) detection and microarray-based comparative genomic hybridisation (array CGH), which may be used to detect copy number aberrations in human tumours. As part of the EuroBoNeT network of excellence for research on bone tumours (eurobonet.eu), we have evaluated four different commercial highresolution microarray platforms in order to identify the most appropriate technology for mapping DNA copy number aberrations in such tumours.
\end{abstract}

Findings: DNA from two different cytogenetically well-characterized bone sarcoma cell lines, representing a simple and a complex karyotype, respectively, was tested in duplicate on four high-resolution microarray platforms; Affymetrix Genome-Wide Human SNP Array 6.0, Agilent Human Genome CGH 244A, Illumina HumanExon510s-duo and Nimblegen HG18 CGH 385 k WG tiling v1.0. The data was analysed using the platform-specific analysis software, as well as a platform-independent analysis algorithm. DNA copy number was measured at six specific chromosomes or chromosomal regions, and compared with the expected ratio based on available cytogenetic information. All platforms performed well in terms of reproducibility and were able to delimit and score small amplifications and deletions at similar resolution, but Agilent microarrays showed better linearity and dynamic range. The platform-specific analysis software provided with each platform identified in general correct copy numbers, whereas using a platform-independent analysis algorithm, correct copy numbers were determined mainly for Agilent and Affymetrix microarrays.

Conclusions: All platforms performed reasonably well, but Agilent microarrays showed better dynamic range, and like Affymetrix microarrays performed well with the platform-independent analysis software, implying more robust data. Bone tumours like osteosarcomas are heterogeneous tumours with complex karyotypes that may be difficult to interpret, and it is of importance to be able to well separate the copy number levels and detect copy number changes in subpopulations. Taking all this into consideration, the Agilent and Affymetrix microarray platforms were found to be a better choice for mapping DNA copy numbers in bone tumours, the latter having the advantage of also providing heterozygosity information.

\section{Background}

Chromosomal aberrations are frequent in cancer, and change in gene dosage is a common mechanism for activation or attenuation of oncogenes and tumour suppressor genes, respectively. In order to precisely identify chromosomal regions of gain and loss, a number of microarraybased technologies have been developed to measure

\footnotetext{
* Correspondence: leonardm@rr-research.no

'Department of Tumour Biology, The Norwegian Radium Hospital, Oslo University Hospital, Oslo, Norway

Full list of author information is available at the end of the article
}

genome-wide DNA copy number [1-3]. Microarray-based comparative genomic hybridisation (array CGH) provides the means of quantitatively measuring DNA copy number aberrations at high-resolution and map them directly to the genome sequence.

High-density oligonucleotide microarrays contain synthetic single-stranded oligonucleotide probes, and different designs for array CGH are offered by a number of companies. The size of the oligonucleotides ranges from 25-mer to 85-mer depending on the type of microarray. Some of these microarrays have been developed for

\section{Ciomed Central}


linkage analysis by the identification of single nucleotide polymorphisms (SNPs), allowing the simultaneous detection of DNA copy number changes and loss of heterozygosity $(\mathrm{LOH})$, which is the regional loss of the contribution to the genome from one parent $[4,5]$. LOH can be copy number neutral when the deleted chromosomal region is compensated by mitotic recombination, resulting in homozygosity without physical DNA loss.

With the increasing number of microarray formats available for detection of DNA copy number changes and $\mathrm{LOH}$, with differences in design, resolution and experimental information obtainable, there is a need to evaluate the alternatives in order to identify the best microarray platform for specific studies. An increasing number of comparative studies of high-resolution platforms have been performed [6-15], addressing different types of research questions. In general, most platforms have been reported to perform well, but differences occur, and obviously each platform has its advantages and disadvantages that need to be taken into consideration.

Benign and malignant bone tumours are often found in bone and show varying degrees of osteogenic, chondrogenic, fibrogenic or neuroectodermal differentiation, among others. Primary malignant bone tumours, or bone sarcomas, arise frequently in the long bones of the extremities [16]. Most tumours arise sporadically, but some occur in relation to pre-existing conditions of bone or inherited diseases. Bone sarcomas can occur at any age, but the tumours frequently occur in children and adolescents in addition to older people. About $35 \%$ of the bone sarcomas are osteosarcomas, $25 \%$ are chondrosarcomas and $16 \%$ are Ewing sarcomas/PNET (Primitive NeuroEctodermal Tumour) [16]. Most conventional osteosarcomas have complex karyotypes with numerous numerical and structural aberrations, whereas the karyotype complexity is in general reduced from chondrosarcomas to Ewing sarcoma/PNET [16]. Both high level of amplification and homozygous deletion of various sizes can occur in these tumour types. Due to their complex karyotype, microarray-based technologies are particularly useful to decipher chromosome aberrations in these tumours.

As part of the EuroBoNeT European network of excellence for research on bone tumours (European Network to Promote Research into Uncommon Cancers in Adults and Children: Pathology, Biology and Genetics of Bone Tumours, eurobonet.eu), we have evaluated four different high-resolution commercial microarray platforms for DNA copy number analysis; Affymetrix Genome-Wide Human SNP Array 6.0, Agilent Human Genome CGH 244A, Illumina HumanExon510s-duo and Nimblegen HG18 CGH 385 k WG tiling v1.0, in order to identify the most appropriate microarray platform for mapping DNA copy number aberrations in bone tumours.

\section{Materials and methods Test material}

Two different cell lines were selected for the testing; TC-32, an Ewing sarcoma/PNET cell line with simple chromosomal aberrations, and OSA (termed SJSA-1 by ATCC), a conventional osteosarcoma cell line with complex genomic rearrangements. The TC-32 cell line was kindly provided by Dr. S.A. Burchill (St. James' University Hospital, Leeds, UK), and the SJSA-1 (OSA) cell line was obtained from ATCC.

TC-32 was cultured in RPMI 1640 GlutaMAX medium supplemented with $20 \%$ foetal calf serum and penicillin/ streptomycin (all Invitrogen, Carlsbad, CA, USA). OSA was grown in RPMI1640 (Lonza, Basel, Switzerland) supplemented with $10 \%$ foetal calf serum (PAA Laboratories $\mathrm{GmbH}$, Pasching, Austria), GlutaMAX (Invitrogen) and penicillin/streptomycin (Lonza), at $37^{\circ} \mathrm{C}$ with $5 \% \mathrm{CO}_{2}$. All cells were split when reaching $80 \%$ confluency.

DNA was isolated from each cell line using the High Pure PCR Template Isolation Kit (Roche, Basel, Switzerland) following the manufacturer's instructions. For two channel arrays (Agilent and Nimblegen), a commercial pooled male DNA was used as a reference (Promega, Madison, WI, USA), while for single channel arrays (Affymetrix and Illumina), data from a normal sample set provided by each supplier was used as a normal reference control. The same DNA preparations from the cell lines, reference DNA and Human Cot-1 DNA (Invitrogen) were used for all platforms and replicates in order to avoid effects of DNA quality or cell line variability on the results.

\section{Microarray platforms}

The cell lines were profiled for DNA copy number changes in duplicate using four different high-resolution oligonucleotide microarray platforms; Affymetrix Genome-Wide Human SNP Array 6.0, Agilent Human Genome CGH 244A, Illumina HumanExon510s-duo and Nimblegen HG18 CGH 385 k WG Tiling v1.0. Information and characteristics for each platform are summarized in Table 1. Hybridisations for Affymetrix and Nimblegen microarrays were carried out at the Microarray Core Facility at The Norwegian Radium Hospital (Oslo, Norway), whereas hybridisations for Agilent and Illumina microarrays were performed at Service XS (Leiden, The Netherlands). For all platforms, the suppliers' protocols were strictly followed. All datasets can be viewed in the microarray depository ArrayExpress (http://www.ebi.ac.uk/microarray-as/ae/, accession number E-TABM-805).

\section{Affymetrix Genome-Wide Human SNP Array 6.0}

The Affymetrix Genome-Wide Human SNP Array 6.0 contains more than 1.8 million genetic markers, including 
Table 1 Microarray platform characteristics

\begin{tabular}{|c|c|c|c|c|c|c|}
\hline Platform & Technology & $\begin{array}{l}\text { Sample } \\
\text { labelling }\end{array}$ & $\begin{array}{l}\text { Sample } \\
\text { requirement }\end{array}$ & $\begin{array}{l}\text { Number of } \\
\text { features }\end{array}$ & $\begin{array}{l}\text { Median probe } \\
\text { spacing }\end{array}$ & Analysis \\
\hline $\begin{array}{l}\text { Affymetrix Genome-Wide Human } \\
\text { SNP Array } 6.0\end{array}$ & $\begin{array}{l}\text { Oligonucleotide } \\
(25 \mathrm{nt})\end{array}$ & $\begin{array}{l}\mathrm{PCR} \\
\text { reduction }\end{array}$ & $0.5 \mu \mathrm{g}$ & $1,852 k$ & $<700 \mathrm{bp}$ & $\begin{array}{l}\text { DNA copy number } \\
\text { and LOH }\end{array}$ \\
\hline $\begin{array}{l}\text { Agilent Human Genome CGH } \\
244 \mathrm{~A}\end{array}$ & $\begin{array}{l}\text { Oligonucleotide } \\
(60 \mathrm{nt})\end{array}$ & $\begin{array}{l}\text { Whole } \\
\text { genome }\end{array}$ & $0.5-1 \mu \mathrm{g}$ & $236 k$ & $8,900 \mathrm{bp}$ & DNA copy number \\
\hline Illumina HumanExon510s-duo & $\begin{array}{l}\text { Oligonucleotide } \\
(25 \mathrm{nt})\end{array}$ & $\begin{array}{l}\mathrm{PCR} \\
\text { reduction }\end{array}$ & $0.75 \mu \mathrm{g}$ & $511 k$ & $3,200 \mathrm{bp}$ & $\begin{array}{l}\text { DNA copy number } \\
\text { and LOH }\end{array}$ \\
\hline $\begin{array}{l}\text { Nimblegen HG18 CGH } 385 \text { k WG } \\
\text { Tiling v1.0 }\end{array}$ & $\begin{array}{l}\text { Oligonucleotide } \\
(50-75 \mathrm{nt})\end{array}$ & $\begin{array}{l}\text { Whole } \\
\text { genome }\end{array}$ & $1 \mu \mathrm{g}$ & $385 k$ & $6,270 \mathrm{bp}$ & DNA copy number \\
\hline
\end{tabular}

Characteristics of the different high-resolution microarray platforms evaluated.

more than 906,000 SNP probes and more than 946,000 probes designed for detection of DNA copy number changes. Quality control and normalization were performed in Genotyping Console v3.0.1 (Affymetrix). Data was quality controlled using the contrast quality control (CQC) algorithm with a minimal call rate of $>86 \%$. DNA copy number analysis was performed in Genotyping Console using quantile probe level normalization, regional GC correction and default settings. Segmentation was performed using an HMM algorithm, and segments were reported as copy number states (i.e. $0,1,2,3$ and $\geq 4$ copies).

\section{Agilent Human Genome CGH 244A}

The Agilent Human Genome CGH 244A microarray contains approximately 236,000 in situ synthesized 60-mer oligonucleotides spanning coding and noncoding regions. Microarray images were processed in Agilent Feature Extraction v9.1 using default settings (no background subtraction and spatial detrend), as well as "ranked consistent probe methods" for normalization. Export files were further analysed using Agilent DNA Analytics v4.0.76. Aberrations were detected using the AMD-2 algorithm with a threshold of 8.0, and a filter against aberrations with less than six probes was used.

\section{Illumina HumanExon510s-duo}

The HumanExon510s-duo microarray is a gene centric array, having the majority of the 511,354 contained markers within or near genes. More than $60 \%$ of the markers are located within $10 \mathrm{~kb}$ of a gene, and the probes provide coverage for $99.9 \%$ of the RefSeq genes. In addition, probes for known regions of copy number variation are included. Fluorescent intensities from the scanner were imported into BeadStudio v3.3.4 (Illumina) and normalized using quantile normalization. DNA copy number analysis was performed using the cnvPartition algorithm v1.2.0 and default settings.

\section{Nimblegen HG18 CGH 385 k WG Tiling v1.0}

The Nimblegen HG18 CGH 385 k WG Tiling v1.0 microarray contains 385,000 long oligonucleotides (50-75-mer) that tile the human genome. Microarray images were processed in NimbleScan v2.3 (Nimblegen), and segmentation was performed using the DNACopy algorithm with default settings.

\section{Platform-independent analysis}

Platform-independent analysis was performed in Nexus (BioDiscovery, El Segundo, CA, USA) using the rank segmentation algorithm with default settings (threshold of 0.6 for high gain, 0.2 for gain, -0.2 for loss and -1.0 for big loss). SNP analysis of the Affymetrix and Illumina data was performed in Nexus using the SNPFASST segmentation algorithm with default settings.

\section{Results and discussion}

Four different commercial high-resolution oligonucleotide microarray platforms, Affymetrix Genome-Wide Human SNP Array 6.0, Agilent Human Genome CGH 244A, Illumina HumanExon510s-duo and Nimblegen HG18 CGH 385 k WG tiling v1.0, were evaluated for genomic profiling of bone tumours. Information and characteristics for each platform are summarized in Table 1. Two different bone sarcoma cell lines, TC-32 (Ewing sarcoma/PNET) and OSA (conventional osteosarcoma), having simple and extensive chromosomal aberrations, respectively, were selected for the testing. Multicolour COBRA-FISH-based karyotyping of the TC32 cell line has been described earlier [17,18], with the resulting karyotype $48, \mathrm{XX},+\mathrm{i}(1)(\mathrm{q} 10),+8, \mathrm{t}(11 ; 22)(\mathrm{q} 24$; q12).

DNA from the cell lines was labelled and hybridised in duplicate to all four microarray types following the manufacturers' instructions, and the same DNA preparations were used for all platforms and replicates in order to avoid effects of DNA quality or cell line variability on the results. Data processing and analysis were performed using the corresponding software for each of the platforms with default settings.

\section{Reproducibility}

To measure the reproducibility of $\log _{2}$ ratios between replicate hybridisations, Pearson's correlation was calculated based on all data points (Table 2). Illumina microarrays showed the highest degree of correlation between 
Table 2 Reproducibility of replicate hybridisations and signal response to copy number

\begin{tabular}{|c|c|c|c|c|c|c|c|c|c|c|}
\hline \multirow[t]{2}{*}{ Platform } & \multirow[t]{2}{*}{$\begin{array}{l}\text { Corr TC- } \\
32\end{array}$} & \multirow[t]{2}{*}{$\begin{array}{l}\text { Corr } \\
\text { OSA }\end{array}$} & \multicolumn{2}{|c|}{$\begin{array}{l}\text { On: CDKN2A } \\
\text { TC-32 }\end{array}$} & \multirow{2}{*}{$\begin{array}{l}\text { 1n: 9p21.3- } \\
\text { p21.2 } \\
\text { TC-32 } \\
\text { Log }_{2} \text { ratio } \\
\text { (SD) }\end{array}$} & \multirow{2}{*}{$\begin{array}{l}\text { 2n: Chr } 2 \\
\text { TC-32 } \\
\text { Log }_{2} \text { ratio } \\
\text { (SD) }\end{array}$} & \multirow{2}{*}{$\begin{array}{l}\text { 3n: Chr } 8 \\
\text { TC-32 } \\
\text { Log }_{2} \text { ratio } \\
\text { (SD) }\end{array}$} & \multirow{2}{*}{$\begin{array}{l}\text { 4n: } 1 \mathrm{q} \\
\text { TC-32 } \\
\text { Log }_{2} \text { ratio } \\
\text { (SD) }\end{array}$} & \multicolumn{2}{|c|}{$\begin{array}{l}\text { >10n: MDM2 } \\
\text { OSA }\end{array}$} \\
\hline & & & $\begin{array}{l}\log _{2} \text { ratio } \\
\text { (SD) }\end{array}$ & $\begin{array}{l}\text { Size } \\
{[k b]}\end{array}$ & & & & & $\begin{array}{l}\log _{2} \text { ratio } \\
\text { (SD) }\end{array}$ & $\begin{array}{l}\text { Size } \\
{[k b]}\end{array}$ \\
\hline Theoretical value & 1.0 & 1.0 & $<-3$ & - & -1.0 & 0.0 & 0.58 & 1.0 & $>3$ & - \\
\hline \multirow{2}{*}{$\begin{array}{l}\text { Affymetrix Genome- } \\
\text { Wide } \\
\text { Human SNP Array } \\
6.0\end{array}$} & 0.75 & 0.84 & $-1.79(0.93)$ & 166 & $-0.53(0.43)$ & $0.00(0.41)$ & $0.34(0.44)$ & $0.56(0.44)$ & $1.86(0.49)$ & 146 \\
\hline & & & $-2.09(0.92)$ & 148 & $-0.62(0.35)$ & $0.00(0.32)$ & $0.37(0.34)$ & $0.59(0.34)$ & $1.99(0.50)$ & 146 \\
\hline \multirow{2}{*}{$\begin{array}{l}\text { Agilent Human } \\
\text { Genome CGH } 244 \mathrm{~A}\end{array}$} & 0.91 & 0.91 & $-4.72(1.57)$ & 135 & $-0.96(0.31)$ & $0.00(0.19)$ & $0.52(0.27)$ & $0.92(0.22)$ & $4.69(0.24)$ & 147 \\
\hline & & & $-4.83(1.53)$ & 135 & $-0.97(0.30)$ & $0.01(0.21)$ & $0.54(0.29)$ & $0.95(0.25)$ & $4.74(0.21)$ & 147 \\
\hline \multirow{2}{*}{$\begin{array}{l}\text { Illumina Human } \\
\text { Exon510 s-duo }\end{array}$} & 0.96 & 0.94 & $-4.72(1.07)$ & 171 & $-0.67(0.54)$ & $-0.05(0.20)$ & $0.22(0.29)$ & $0.36(0.26)$ & $1.24(0.59)$ & 122 \\
\hline & & & $-4.69(0.91)$ & 171 & $-0.67(0.59)$ & $-0.05(0.20)$ & $0.22(0.29)$ & $0.36(0.26)$ & $1.23(0.58)$ & 122 \\
\hline \multirow{2}{*}{$\begin{array}{l}\text { Nimblegen HG18 } \\
\text { CGH } \\
385 \text { k WG Tiling } \\
\text { v1.0 }\end{array}$} & 0.63 & 0.73 & $-0.85(0.57)$ & 169 & $-0.45(0.30)$ & $-0.06(0.19)$ & $0.24(0.21)$ & $0.48(0.24)$ & $2.58(0.98)$ & 156 \\
\hline & & & $-0.88(0.50)$ & 169 & $-0.47(0.29)$ & $-0.06(0.20)$ & $0.26(0.21)$ & $0.53(0.23)$ & $2.46(1.0)$ & 156 \\
\hline
\end{tabular}

Pearson's correlation of $\log _{2}$ ratios for the replicate hybridisations based on all data points and the average log 2 ratio with standard deviation for six specific chromosomes or chromosomal regions, representing 0,1, 2, 3 and 4 copies in TC-32, as well as high-level amplification in OSA, for each hybridisation. In addition, the measured size of the regions for homozygous deletion of the CDKN2A locus in TC-32 and high-level amplification of the MDM2 locus in OSA is given for each hybridisation.

replicates, 0.96 and 0.94 for $\mathrm{TC}-32$ and OSA, respectively. Agilent microarrays showed also a high correlation between replicates, 0.91 for both cell lines, while Affymetrix microarrays showed an intermediate correlation of 0.75 and 0.84 for TC-32 and OSA, respectively. Nimblegen microarrays showed the lowest correlation, 0.73 and 0.63 for OSA and $\mathrm{TC}-32$, respectively. Although having a more complex karyotype, the OSA cell line showed slightly better correlation for the replicates than the TC-32 cell line for Affymetrix and Nimblegen microarrays. Scatter plots of $\log _{2}$ ratios for the replicate hybridisations for all microarray platforms are shown in Additional file 1.

In a previous study of melanoma cell lines using lower resolution oligonucleotide microarrays from Affymetrix, Agilent and Nimblegen, similar results were observed [8]. Here, the highest resolution array from Agilent tested (185 $\mathrm{K})$ showed the highest correlations (ranging 0.72-0.86 for the different samples hybridised), whereas the highest resolution array from Affymetrix tested (500 K) showed intermediate correlations (0.54-0.67). Also here the Nimblegen array $(1500 \mathrm{~K})$ showed the lowest correlations (0.27-0.57). In addition, a significant higher degree of correlation was observed for the higher-density microarrays compared to the lower-density microarrays from the same supplier (Agilent $185 \mathrm{~K}$ vs $44 \mathrm{~K}$ and Affymetrix $500 \mathrm{~K}$ vs $100 \mathrm{~K})$ [8]. Although not directly comparable, this is in concordance with the even higher correlations between replicate hybridisations observed with the higher-resolution microarrays from Agilent and Affymetrix used here.

Similar results have also been observed between replicate hybridisations of Affymetrix $500 \mathrm{~K}$ arrays, Agilent 44B arrays and Illumina Hap550 arrays for one leukaemia cell line [14]. The standard deviation for each probe across four replicate hybridisations was calculated, and Illumina arrays showed the lowest median standard deviation (0.059), followed by Agilent (0.083) and Affymetrix (0.101). However, when the median standard deviation was normalized to the number of measurements on each platform, all platforms showed similar levels of variation [14].

\section{Signal response to copy number}

To quantify and evaluate the signal response to copy number for each platform, the measured DNA copy numbers for five specific chromosomes or chromosomal regions in TC-32 (representing 0, 1, 2, 3 and 4 copies) and one specific chromosomal region in OSA (representing high-level amplification) were compared with the expected ratios based on available cytogenetic and molecular information. Homozygous deletion of the locus around the tumour suppressor gene CDKN2A in $9 \mathrm{p} 21.3$, heterozygous deletion of 9p21.3-p21.2, normal copy number of chromosome 2, three copies of 
chromosome 8 and four copies of the long arm of chromosome 1 were measured in TC-32, and high-level amplification of the locus around the oncogene $M D M 2$ in 12 q15 was measured in OSA. For both replicate hybridisations, the average $\log _{2}$ ratio was determined for each normalized segment, as well as the standard deviation of the signals, and this is presented in Table 2. Figure 1 shows a plot of the measured average $\log _{2}$ ratios compared to the theoretical values. In addition, the size of the homozygous deletion of the CDKN2A locus in TC-32 and the high-level amplification of the MDM2 locus in OSA were estimated (Table 2). Copy number plots of the homozygous deletion of the $C D K N 2 A$ locus as well as the heterozygous deletion of 9p21.3-p21.2 in TC-32 are shown in Additional file 2 for all microarray platforms.

The values were in general similar between the replicate hybridisations for all platforms and regions. Agilent arrays showed the highest dynamic range, from average $\log _{2}$ ratio of -4.78 to 4.72 for the homozygous deletion of the CDKN2A locus and the high-level amplification of the $M D M 2$ locus, respectively, and gave almost expected $\log _{2}$ ratios for all regions, close to the theoretical values (Table 2). Affymetrix results were second best for $\log _{2}$ ratios for regions of increased copy number, 3 and 4 copies, but not so good on regions of decreased copy number, 0 and 1 copies. The Illumina results deviated most from the expected $\log _{2}$ ratios for 3 and 4 copies (theoretical value 0.58 and 1.0, respectively), giving on average $\log _{2}$ ratios of 0.22 and 0.36 , respectively, while the Nimblegen data deviated most for 0 and 1 copies (theoretical value $<-1$ and -1.0 , respectively), giving on average $\log _{2}$ ratios of -0.87 and -0.46 , respectively. For the highlevel amplification of the MDM2 locus in OSA, expected to have a $\log _{2}$ ratio well above 3 based on previous results $[19,20]$, Nimblegen arrays gave the second best values, average $\log _{2}$ ratio of 2.52 , whereas Affymetrix and Illumina arrays gave average $\log _{2}$ ratios of 1.93 and 1.24 , respectively.

A regression line was calculated for the measured average $\log _{2}$ ratios of the regions representing $1,2,3$ and 4 copies, and the slope and $R^{2}$ values are given in Figure 1 . The regions representing 0 and $>10$ copies were omitted from the regression line, since the expected $\log _{2}$ ratio for the homozygous deletion is not an exact number $(<-3)$ and the exact $\log _{2}$ ratio for the high-level amplification is unknown (>3). All platforms showed a high linearity of the measured $\log _{2}$ ratios, but the slope of the regression line varied. The slope of the measurements for the Agilent arrays was closest to the theoretical value, followed by Affymetrix and Illumina arrays, whereas the measurements from the Nimblegen arrays deviated most.
In a previous study using lower resolution oligonucleotide microarrays from Affymetrix (250 K), Agilent $(185 \mathrm{~K})$ and Illumina $(317 \mathrm{~K})$, as well as BAC arrays (32 K), for screening chronic lymphocytic leukaemia, similar results were observed [9]. Agilent $185 \mathrm{~K}$ arrays and $32 \mathrm{~K} \mathrm{BAC}$ arrays showed the highest dynamic range, where the Agilent arrays showed the most correct response to loss of one copy and gain of one copy, whereas the BAC arrays showed the most correct response to homozygous deletion. A notable difference in the scale of $\log _{2}$ ratios has also previously been observed between Agilent 44 K arrays, Illumina $109 \mathrm{~K}$ arrays and ROMA/Nimblegen $82 \mathrm{~K}$ arrays for screening breast cancer, with higher signals for the Agilent arrays [6]. Affymetrix $100 \mathrm{~K}$ and $250 \mathrm{~K}$ arrays also showed higher mean $\log _{2}$ ratio of chromosome $\mathrm{X}$ than Nimblegen $385 \mathrm{~K}$ arrays in sex-mismatched hybridisations of patients with submicroscopic genomic copy number variations [11]. In a previous study on melanoma cell lines, Agilent $185 \mathrm{~K}$ arrays showed the highest signals for 4 copies (average $\log _{2}$ ratio 0.86 ) as well as the highest signal to noise ratio, whereas Affymetrix $500 \mathrm{~K}$ arrays showed intermediate values (average 0.55) and Nimblegen $1500 \mathrm{~K}$ arrays showed the lowest values (average 0.37) [8].

Concerning the variation in $\log _{2}$ ratios within a chromosome or chromosomal region, Affymetrix arrays showed the highest standard deviation for the regions

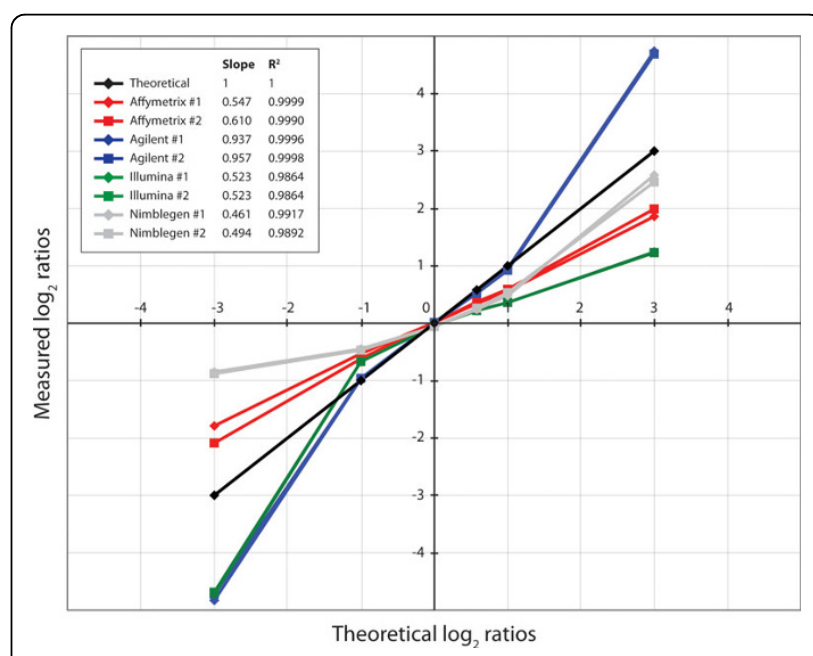

Figure 1 Plot of measured $\log _{2}$ ratios compared to theoretical values for specific chromosomes or chromosomal regions. Plot of average measured $\log _{2}$ ratios compared to theoretical values for six specific chromosomes or chromosomal regions; the CDKN2A locus in 9p21.3, 9p21.3-p21.2, chromosome 2, chromosome 8, 1q (all in TC-32) and the MDM2 locus in 12q15 (in OSA) (representing 0, 1, 2, 3, 4 and $>10$ copies, respectively) for all microarray platforms and replicates. The slope and regression coefficient $\left(R^{2}\right)$ for the regression line based on four of the regions, representing 1, 2, 3 and 4 copies, are given. 
representing 2, 3 and 4 copies, whereas the other platforms showed equal variation. Illumina and Agilent arrays showed the highest standard deviation for the regions representing 1 and 0 copies, respectively (0.57 and 1.55). Nimblegen arrays showed the lowest standard deviations for the regions of loss, but this is most likely due to compression of the $\log _{2}$ ratios since the Nimblegen data deviated most for 0 and 1 copies. For the high-level amplification of the MDM2 locus, Agilent showed the lowest and Nimblegen the highest standard deviations (0.23 and 0.99 , respectively).

The baseline variation has also been determined in previous studies. For the screening of chronic lymphocytic leukaemia, Affymetrix $250 \mathrm{~K}$ arrays, Agilent $185 \mathrm{~K}$ arrays and Illumina $317 \mathrm{~K}$ arrays showed similar average $\log _{2}$ ratio and standard deviation of a region with normal copy number (chromosome 1) [9]. However, when assessing the baseline variation in form of autocorrelation of the whole genome, Agilent showed the lowest variation, followed by Affymetrix and Illumina. For the previous study of patients with submicroscopic genomic copy number variations, Nimblegen $185 \mathrm{~K}$ arrays showed a higher standard deviation of the $\log _{2}$ ratios of the whole genome (excluding regions harbouring the variations) than the Affymetrix $250 \mathrm{~K}$ and $100 \mathrm{~K}$ arrays [11].

The distribution of $\log _{2}$ ratios of all probes within the six specific chromosomes or chromosomal regions (representing $0,1,2,3,4$ and $>10$ copies, respectively) for one hybridisation from all microarray platforms is shown in Figure 2. The replicate hybridisation showed a similar pattern (data not shown). The distribution is shown for both the normalized data and a smoothed version of the same data. The data were smoothed using Gaussian smoothing with a window size of $50 \mathrm{~kb}$ and standard deviation of $10 \mathrm{~kb}$, in order to reduce the variation. Smoothing was not possible for the small regions representing 0 and $>10$ copies (the CDKN2A and $M D M 2$ loci) due to insufficient number of probes. For the normalized data, the curves for the different copy number levels were by far best separated by the Agilent data, whereas the curves were highly overlapping for the Affymetrix data. However, smoothing of the data had a huge effect on the Affymetrix data, narrowing the distributions of the $\log _{2}$ ratios and thus better separating the curves. The smoothing also further improved the Agilent, Illumina and Nimblegen data, but to a smaller extent. The most difficult separation for all platforms was to distinguish between 3 and 4 copies, and this was particularly not easy with the Illumina data where the two curves were highly overlapping.

Similar results were observed in a previous study of melanoma cell lines, with the distribution of $\log _{2}$ ratios of regions of 2 and 4 copies [8]. The distribution was best separated for the Agilent $185 \mathrm{~K}$ and $44 \mathrm{~K}$ arrays, whereas the Affymetrix $500 \mathrm{~K}$ and $100 \mathrm{~K}$ arrays showed intermediate results. For the Nimblegen $1500 \mathrm{~K}$ arrays, the distribution of $\log _{2}$ ratios of regions of 2 and 4 copies was indistinguishable. However, in line with the observations in this study, smoothing of the data improved the results for all platforms, with a most profound effect for the Nimblegen $1500 \mathrm{~K}$ arrays and Affymetrix $100 \mathrm{~K}$ arrays, the two lowest ranking in terms of signal to noise ratios [8].

The size of the small aberrations of $C D K N 2 A$ and $M D M 2$ was estimated manually, and this revealed very similar results for all microarray platforms (Table 2). Only Affymetrix arrays showed a difference in size of the deletion of the $C D K N 2 A$ locus between the replicate hybridisations for TC-32, detected to be 166 and $148 \mathrm{~kb}$, respectively.

\section{Detection of DNA copy number aberrations}

The aberrant regions examined for signal response to copy number were scored using the analysis software provided for each microarray platform with default settings. In addition, data from all platforms were exported into Nexus (BioDiscovery) in order to make an independent scoring of the aberrations. This software also has advantages when it comes to downstream integration with other genome-level data. In Nexus, all four platforms were analysed using the rank segmentation algorithm with default settings. Table 3 shows the scoring of the aberrant regions examined for signal response to copy number from the platform-specific analysis software as well as Nexus for one hybridisation from all platforms. The replicate hybridisation showed a similar pattern (data not shown). Detection of the homozygous deletion of the CDKN2A locus as well as the heterozygous deletion of 9p21.3-p21.2 in TC-32 using Nexus for all platforms is shown in Additional file 3.

Using the platform-specific analysis software, Affymetrix, Agilent and Illumina scored the correct copy number level for all regions representing $0,1,2,3$ and 4 copies in $\mathrm{TC}-32$, and indicated the high-level amplification in OSA. The only exception was the scoring of 4 copies of 1q using the Illumina data, where the software segmented the region into segments of mainly 3 copies and some smaller segments of 4 copies. For Nimblegen, the corresponding analysis software segments the data and displays them, without giving copy number scores.

Using the platform-independent Nexus software, all regions were determined to have the correct copy number for the Affymetrix data (Table 3). The region of 1 copy was over-scored as a homozygous deletion for Agilent, because of the $\operatorname{low} \log _{2}$ ratios of this segment (average -0.97) and the threshold for homozygous deletion in Nexus (default $\log _{2}$ ratio $<-1.0$ ). The region of 4 


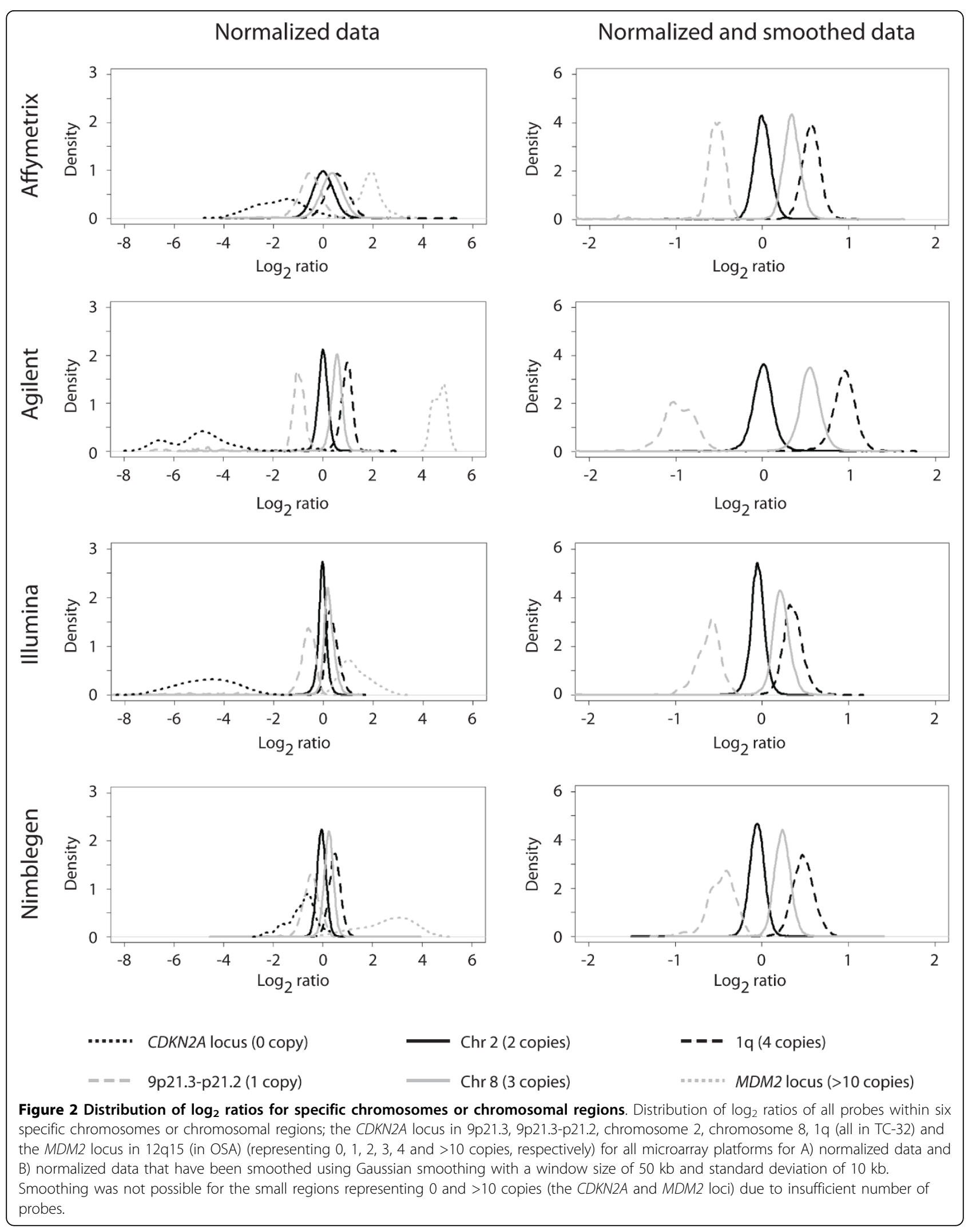


Table 3 Detection of copy number of specific regions

\begin{tabular}{|c|c|c|c|c|c|c|c|c|c|c|}
\hline \multirow[t]{2}{*}{ Platform } & \multicolumn{2}{|c|}{$\begin{array}{l}\text { 0n: CDKN2A } \\
\text { TC-32 }\end{array}$} & \multicolumn{2}{|c|}{$\begin{array}{l}\text { 1n: 9p21.3-p21.2 } \\
\text { TC-32 }\end{array}$} & \multicolumn{2}{|l|}{$\begin{array}{l}\text { 3n: Chr } 8 \\
\text { TC-32 }\end{array}$} & \multicolumn{2}{|l|}{$\begin{array}{l}\text { 4n: } 1 \mathrm{q} \\
\text { TC-32 }\end{array}$} & \multicolumn{2}{|c|}{$\begin{array}{l}\text { >10n: MDM2 } \\
\text { OSA }\end{array}$} \\
\hline & Platform & Nexus & Platform & Nexus & Platform & Nexus & Platform & Nexus & Platform & Nexus \\
\hline Affymetrix Genome-Wide Human SNP array 6.0 & Yes & Yes & Yes & Yes & Yes & Yes & Yes & Yes & Yes & Yes \\
\hline Agilent Human Genome CGH 244A & Yes & Yes & Yes & On & Yes & Yes & Yes & Yes & Yes & Yes \\
\hline Illumina HumanExon510s-duo & Yes & Yes & Yes & Yes & Yes & Yes & $3 n / 4 n$ & $3 n$ & Yes & Yes \\
\hline Nimblegen HG18 CGH 385 k WG Tiling v1.0 ${ }^{1}$ & - & $1 \mathrm{n}$ & - & Yes & - & Yes & - & $3 n$ & - & Yes \\
\hline
\end{tabular}

${ }^{1}$ Nimblegen's analysis software segments the data without scoring of copy number

Detection of copy number of five specific chromosomes or chromosomal regions representing 0, 1, 3 and 4 copies in TC-32, as well as high-level amplification in OSA, using the platform-specific software and the platform-independent software Nexus.

copies was scored as one copy less for Illumina, most likely due to compression of the $\log _{2}$ values. For the Nimblegen data, Nexus detected the homozygous deletion as 1 copy and the 1q region as 3 copies instead of 4 , most likely also due to compression of the $\log _{2}$ values.

In a previous study, Affymetrix $500 \mathrm{~K}$ arrays, Agilent $244 \mathrm{~K}$ arrays and Nimblegen $385 \mathrm{~K}$ arrays were compared for detection of submicroscopic constitutional aberrations [15]. In that study, using the corresponding analysis program, all 10 previously known abnormalities investigated were detected using the Agilent data, whereas one and three aberrations were not identified using the Affymetrix and Nimblegen data, respectively. However, using the software dChip in combination with an R script, all aberrations were detected using the Affymetrix data as well [15]. For the comparison of Affymetrix $500 \mathrm{~K}$ arrays, Agilent 44B arrays and Illumina Hap550 arrays, all known alterations in a leukaemia cell line were identified using both a platform-specific software and a platform-independent analysis (circular binary segmentation) [14].

The number of overall copy number aberrations in chromosome 1-22 detected by Nexus is given in Table 4, for both TC-32 and OSA for all microarray platforms and both replicate hybridisations. Detection of the copy number aberrations is given in Additional File 4. Nexus divides the copy number aberrations in four categories; homozygous copy loss, loss, gain and high copy gain depending on the $\log _{2}$ ratio of the segments. The number of detected copy number aberrations was in general similar between the replicate hybridisations, except for the categories gain and loss in TC-32 by Affymetrix, where the replicates varied with 31 and 36 aberrations, respectively.

The number of detected aberrations varied between the platforms, in general showing that microarrays with a higher number of probes detect more segments of copy number aberrations. Affymetrix showed by far the highest number of aberrations, as expected with the 1.8 million probes on the array, and most additional aberrations compared to the other platforms were small regions (Table 4 and Additional File 4). However, Agilent, with only $236 \mathrm{k}$ probes on the array, also showed a high number of small aberrations in the OSA cell line. Illumina identified approximately the same number of total aberrations as Agilent, whereas Nimblegen identified considerably less than the other platforms (Table 4). Some differences between the platforms were observed for larger regions, for instance the heterozygous deletion of $4 \mathrm{q}$ in OSA detected by Agilent, which was partly detected by

Table 4 Detection of copy number aberrations

\begin{tabular}{|c|c|c|c|c|c|c|c|c|}
\hline \multirow[t]{2}{*}{ Platform } & \multicolumn{4}{|c|}{ TC-32 } & \multicolumn{4}{|c|}{ OSA } \\
\hline & $\begin{array}{l}\text { Homozygous } \\
\text { copy loss }\end{array}$ & Loss & Gain & High copy gain & $\begin{array}{l}\text { Homozygous } \\
\text { copy loss }\end{array}$ & Loss & Gain & $\begin{array}{l}\text { High copy } \\
\text { gain }\end{array}$ \\
\hline \multirow[t]{2}{*}{ Affymetrix Genome-Wide Human SNP array 6.0} & 19 & 45 & 40 & 21 & 18 & 100 & 101 & 72 \\
\hline & 23 & 76 & 76 & 28 & 16 & 114 & 123 & 82 \\
\hline \multirow[t]{2}{*}{ Agilent Human Genome CGH 244A } & 4 & 11 & 10 & 2 & 9 & 82 & 58 & 42 \\
\hline & 4 & 8 & 10 & 2 & 13 & 103 & 65 & 37 \\
\hline \multirow[t]{2}{*}{ Illumina HumanExon510s-duo } & 3 & 32 & 21 & 3 & 2 & 75 & 77 & 33 \\
\hline & 3 & 33 & 19 & 2 & 4 & 86 & 78 & 36 \\
\hline \multirow{2}{*}{$\begin{array}{l}\text { Nimblegen HG18 CGH } 385 \text { k } \\
\text { WG Tiling v1.0 }\end{array}$} & 0 & 7 & 15 & 2 & 1 & 31 & 62 & 36 \\
\hline & 0 & 11 & 6 & 0 & 1 & 25 & 55 & 36 \\
\hline
\end{tabular}

Number of copy number aberrations detected in chromosome 1-22 in TC-32 and OSA using the platform-independent software Nexus. 
Illumina and not at all by Nimblegen. Affymetrix detected several small regions within $4 \mathrm{q}$ as deletions (Additional File 4).

Similar results were observed in the analysis of the total number of chromosome segments altered in a leukaemia cell line, where the highest resolution arrays (Illumina Hap550) showed the highest number of identified segments, followed by Affymetrix $500 \mathrm{~K}$ arrays and Agilent $44 \mathrm{~B}$ arrays [14]. On the other hand, for the screening of chronic lymphocytic leukaemia, the lowest resolution array (Agilent $185 \mathrm{~K}$ ) detected the highest number of platform-specific copy number aberrations, followed by Affymetrix $250 \mathrm{~K}$ arrays and Illumina $317 \mathrm{~K}$ arrays [9]. Most of these aberrations were smaller segments. For the aberrations detected in common by two of the platforms, most often the Affymetrix and Agilent platforms showed concordant results [9]. A comparison of copy number aberrations detected in 18 melanoma cell lines by Affymetrix $500 \mathrm{~K}$ arrays and Agilent $244 \mathrm{~K}$ arrays showed a similar number of total aberrations detected with a $29 \%$ overlap between the two platforms [8].

\section{Scoring of loss of heterozygosity}

An advantage of the Affymetrix and Illumina platforms is that they also provide global polymorphism information, and thus can indicate regions of $\mathrm{LOH}$ that could be involved in loss-of-function mutations, haploinsufficiency, etc. SNP analysis of the Affymetrix and Illumina data was performed in Nexus using the SNP-FASST segmentation algorithm with default settings.

In general, Affymetrix detected slightly more regions of allelic changes overall for both samples, but both platforms detected allelic changes in the regions with copy number aberrations scored by Nexus. In addition, regions of copy number-neutral allelic changes were identified, and detection of the copy number-neutral LOH of 1q in OSA using Nexus is shown in Additional file 5. Nexus divides the detection of allelic changes in two categories; $\mathrm{LOH}$ and allelic imbalance, depending on the distribution of the allelic ratio plot. For the copy-number neutral LOH of $1 \mathrm{q}$ in OSA, as well as other similar regions, the allelic changes were scored as $\mathrm{LOH}$ for the Illumina data, whereas the allelic changes were only scored as allelic imbalance for the Affymetrix data, due to a less defined distribution of the allelic ratio plot. The allelic ratios of the Illumina SNP data were overall better separated and thus more precisely scored, but all the allelic changes identified using the Illumina data were also identified using the Affymetrix data. Thus, the two platforms both perform well in detecting regions of allelic imbalance based on the SNP data.

Detection of LOH has previously been compared for Affymetrix $250 \mathrm{~K}$ arrays and Illumina $317 \mathrm{~K}$ arrays for chronic lymphocytic leukaemia [9]. Most loci were concordant between the two platforms, especially for regions $>4 \mathrm{Mb}$, but more differences were observed for smaller regions. The Illumina arrays showed in general a higher detection rate, in contrast with this study, but also a lower noise level in the $\mathrm{LOH}$ analysis, which was also observed in this study.

\section{Conclusions}

All microarray platforms performed well in terms of reproducibility, delimiting small amplifications and deletions, as well as scoring the aberrations. Agilent microarrays showed better linearity and dynamic range since the measured $\log _{2}$ ratios using these platforms were closer to the expected ratios. The platform-specific analysis software identified in general correct copy numbers, whereas using a platform-independent analysis algorithm, correct copy numbers were determined mainly for Agilent and Affymetrix microarrays, implying more robust data. Bone tumours like osteosarcomas are heterogeneous tumours with complex karyotypes that may be difficult to interpret, and it is of importance to be able to well separate the copy number levels and detect copy number changes in subpopulations. Less complex tumours will also benefit from an increased linearity and dynamic range by allowing reliable detection of small subpopulations of cells with DNA copy number changes within relatively homogenous tumours. Taking all this into consideration, the Agilent and Affymetrix microarray platforms were found to be a better choice for mapping DNA copy numbers in bone tumours, the latter having the advantage of also providing heterozygosity information.

\section{Additional material}

Additional file 1: Scatter plot of $\log _{2}$ ratios and the correlation coefficient for the replicate hybridisations of TC-32 and OSA for all microarray platforms.

Additional file 2: Copy number plot of the homozygous deletion of the CDKN2A locus and the heterozygous deletion of 9p21.3-p21.2 in TC-32 for all microarray platforms.

Additional file 3: Detection of the homozygous deletion of the CDKN2A locus and the heterozygous deletion of 9p21.3-p21.2 in TC-32 using Nexus for all microarray platforms.

Additional file 4: Detection of copy number aberrations in chromosome 1-22 in TC-32 and OSA using Nexus for all microarray platforms

Additional file 5: Detection of the copy number-neutral LOH of 1q in OSA using Nexus for the Affymetrix and Illumina platforms.

\section{Acknowledgements}

We would like to thank the personnel at the Microarray Core Facility at The Norwegian Radium Hospital (Oslo, Norway) and Service XS (Leiden, The Netherlands) for technical assistance. This work was supported by the European Network to Promote Research into Uncommon Cancers in Adults and Children: Pathology, Biology and Genetics of Bone Tumours 
(EuroBoNeT), the Norwegian Cancer Society and the FUnctional GEnomics programme (FUGE) of the Research Council of Norway.

\section{Author details}

${ }^{1}$ Department of Tumour Biology, The Norwegian Radium Hospital, Oslo University Hospital, Oslo, Norway. '2Department of Molecular Cell Biology, Leiden University Medical Center, Leiden, The Netherlands. ${ }^{3}$ Norwegian Microarray Consortium, Department of Molecular Biosciences, University of Oslo, Oslo, Norway. ${ }^{4}$ Department of Informatics, University of Oslo, Oslo, Norway. ${ }^{5}$ Department of Pathology, Leiden University Medical Center, Leiden, The Netherlands.

\section{Authors' contributions}

SHK participated in the microarray experiments and analyses and drafted the manuscript, KS participated in the microarray analyses, conceived of the study, participated in its design and coordination, AHBP participated in the microarray experiments, HR participated in the microarray analyses, AMCJ conceived of the study, OM conceived of the study, participated in its design and coordination and helped to draft the manuscript, LAMZ carried out the microarray analyses, conceived of the study, participated in its design and coordination and helped to draft the manuscript. All authors read and approved the final manuscript.

\section{Competing interests}

The authors declare that they have no competing interests.

Received: 12 May 2010 Accepted: 8 August 2010

Published: 8 August 2010

\section{References}

1. Pinkel D, Segraves R, Sudar D, Clark S, Poole I, Kowbel D, Collins C, Kuo WL, Chen $C$, Zhai $Y$, et al: High resolution analysis of DNA copy number variation using comparative genomic hybridization to microarrays. Nat Genet 1998, 20(2):207-211.

2. Pollack JR, Perou CM, Alizadeh AA, Eisen MB, Pergamenschikov A Williams CF, Jeffrey SS, Botstein D, Brown PO: Genome-wide analysis of DNA copy-number changes using cDNA microarrays. Nat genet 1999, 23(1):41-46.

3. Solinas-Toldo S, Lampel S, Stilgenbauer S, Nickolenko J, Benner A, Dèohner $H$, Cremer T, Lichter P: Matrix-based comparative genomic hybridization: biochips to screen for genomic imbalances. Genes Chromosomes Cancer 1997, 20(4):399-407.

4. Bignell GR, Huang J, Greshock J, Watt S, Butler A, West S, Grigorova M, Jones KW, Wei W, Stratton MR, et al: High-resolution analysis of DNA copy number using oligonucleotide microarrays. Genome Res 2004, 14:287-295.

5. Zhou X, Rao NP, Cole SW, Mok SC, Chen Z, Wong DT: Progress in concurrent analysis of loss of heterozygosity and comparative genomic hybridization utilizing high density single nucleotide polymorphism arrays. Cancer Genet Cytogenet 2005, 159:53-57.

6. Baumbusch LO, Aaroe J, Johansen FE, Hicks J, Sun H, Bruhn L, Gunderson K, Naume B, Kristensen VN, Liestol K, et al: Comparison of the Agilent, ROMA/NimbleGen and Illumina platforms for classification of copy number alterations in human breast tumors. BMC Genomics 2008, 9:379.

7. Coe BP, Ylstra B, Carvalho B, Meijer GA, Macaulay C, Lam WL: Resolving the resolution of array CGH. Genomics 2007, 89:647-653.

8. Greshock J, Feng B, Nogueira C, Ivanova E, Perna I, Nathanson K, Protopopov A, Weber BL, Chin L: A comparison of DNA copy number profiling platforms. Cancer Res 2007, 67:10173-10180.

9. Gunnarsson R, Staaf J, Jansson M, Ottesen AM, Goransson H, Liljedahl U, Ralfkiaer U, Mansouri M, Buhl AM, Smedby KE, et al: Screening for copynumber alterations and loss of heterozygosity in chronic lymphocytic leukemia-a comparative study of four differently designed, high resolution microarray platforms. Genes Chromosomes Cancer 2008, 47:697-711.

10. Gaasenbeek M, Howarth K, Rowan AJ, Gorman PA, Jones A, Chaplin T, Liu Y, Bicknell D, Davison EJ, Fiegler $H$, et al: Combined array-comparative genomic hybridization and single-nucleotide polymorphism-loss of heterozygosity analysis reveals complex changes and multiple forms of chromosomal instability in colorectal cancers. Cancer Res 2006, 66:3471-3479.
11. Hehir-Kwa JY, Egmont-Petersen M, Janssen IM, Smeets D, van Kessel AG, Veltman JA: Genome-wide copy number profiling on high-density bacterial artificial chromosomes, single-nucleotide polymorphisms, and oligonucleotide microarrays: a platform comparison based on statistical power analysis. DNA Res 2007, 14:1-11.

12. Kloth JN, Oosting J, van Wezel T, Szuhai K, Knijnenburg J, Gorter A, Kenter GG, Fleuren GJ, Jordanova ES: Combined array-comparative genomic hybridization and single-nucleotide polymorphism-loss of heterozygosity analysis reveals complex genetic alterations in cervical cancer. BMC Genomics 2007, 8:53.

13. Wicker N, Carles A, Mills IG, Wolf M, Veerakumarasivam A, Edgren $H$, Boileau F, Wasylyk B, Schalken JA, Neal DE, et al: A new look towards BACbased array $\mathrm{CGH}$ through a comprehensive comparison with oligobased array CGH. BMC Genomics 2007, 8:84.

14. Hester SD, Reid L, Nowak N, Jones WD, Parker JS, Knudtson K, Ward W, Tiesman J, Denslow ND: Comparison of comparative genomic hybridization technologies across microarray platforms. J Biomol Tech 2009, 20:135-151.

15. Zhang ZF, Ruivenkamp C, Staaf J, Zhu H, Barbaro M, Petillo D, Khoo SK, Borg A, Fan YS, Schoumans J: Detection of submicroscopic constitutional chromosome aberrations in clinical diagnostics: a validation of the practical performance of different array platforms. Eur J Hum Genet 2008, 16:786-792.

16. Fletcher CDM, Unni KK, Mertens F: World Health Organization Classification of Tumours. Pathology and Genetics of Tumours of Soft Tissue and Bone Lyon: IARC Press 2002.

17. Szuhai K, ljszenga M, Tanke HJ, Rosenberg C, Hogendoorn PC: Molecular cytogenetic characterization of four previously established and two newly established Ewing sarcoma cell lines. Cancer Genet Cytogenet 2006, 166:173-179.

18. Szuhai K, Tanke HJ: COBRA: combined binary ratio labeling of nucleicacid probes for multi-color fluorescence in situ hybridization karyotyping. Nat Protoc 2006, 1:264-275.

19. Henriksen J, Aagesen TH, Maelandsmo GM, Lothe RA, Myklebost O, Forus A: Amplification and overexpression of COPS3 in osteosarcomas potentially target TP53 for proteasome-mediated degradation. Oncogene 2003, 22:5358-5361.

20. Ottaviano L, Schaefer KL, Gajewski M, Huckenbeck W, Baldus S, Rogel U, Mackintosh C, de Alava E, Myklebost O, Kresse SH, et al: Molecular characterization of commonly used cell lines for bone tumor research: a trans-European EuroBoNet effort. Genes Chromosomes Cancer 2010, 49:40-51.

doi:10.1186/1756-0500-3-223

Cite this article as: Kresse et al.: Evaluation of high-resolution microarray platforms for genomic profiling of bone tumours. BMC Research Notes $20103: 223$

\section{Submit your next manuscript to BioMed Central and take full advantage of:}

- Convenient online submission

- Thorough peer review

- No space constraints or color figure charges

- Immediate publication on acceptance

- Inclusion in PubMed, CAS, Scopus and Google Scholar

- Research which is freely available for redistribution 